\title{
Natural Numbers Applied to Physical Constants: Spacetime Discreteness
}

\author{
Alberto Coe \\ Independent Researcher, Oviedo, Spain \\ Email: albamv8@gmail.com
}

How to cite this paper: Coe, A. (2017) Natural Numbers Applied to Physical Constants: Spacetime Discreteness. Journal of Modern Physics, 8, 17-23.

http://dx.doi.org/10.4236/jmp.2017.81002

Received: December 6, 2016

Accepted: December 25, 2016

Published: December 28, 2016

Copyright $\odot 2017$ by author and Scientific Research Publishing Inc. This work is licensed under the Creative Commons Attribution International License (CC BY 4.0).

http://creativecommons.org/licenses/by/4.0/

\begin{abstract}
We analyze some physical concepts only using natural numbers. We assume a discrete space. Physical variables such as speed and momentum are considered as result of the sum of discrete contributions. Such discrete contributions can be calculated with natural numbers only. Elementary algebra is used in the analysis of physical subjects.
\end{abstract}

\section{Keywords}

Space-Time Discreteness, Natural Numbers

\section{Introduction}

In his book "The Road to Reality: A Complete Guide to the Laws of the Universe" [1], Roger Penrose devotes some pages to the relationship between the physics and the natural numbers. Penrose asks: Do natural numbers need the physical world? Although at a purely algebraic level, arithmetical level (for more information), we explore the use of natural numbers in the field of Physics. In other words (paraphrasing Penrose): do some physical phenomena need the use of natural numbers?

Discreteness of spacetime is based on a physical postulate, i.e. the existence of a minimum interval of spacetime. In the microphysical realm you must do many measurements about the position or speed of a particle. As the uncertainty principle stated by Heisenberg, you cannot know both variables at the same time with accuracy. For that it's worth to articulate the measurements in terms of probability in the position and momentum of a particle.

So the significance of this paper has to do with the branch of Physics that analyzes the nature of the reality under a discrete point of view. We explore briefly the relationship between several physical topics with the so-called natural numbers. On the one hand, we follow the theory that assumes the discreteness of spacetime. On the other hand, we put into practice an analysis of such discrete nature of the physical world by means of series of natural numbers. 


\section{Method}

DISCRETE CONTRIBUTION SERIES (dics):

Define dics as a sum of natural numbers that show a peculiar sequence, for instance series that has a symmetric appearance like

$$
a=(1+2+16+32+16+2+1) .
$$

Other times will apply this kind of series

$$
b=(1+2+16+32) \text {. }
$$

When has been removed some numbers from the series $a$.

Often will write a series multiplied by other numbers, for instance

$$
b=(1+2+16+32) \times 10^{n} \times 2^{m}
$$

where $n$ and $m$ are integer numbers.

It's worth noting that the symbols used by means of lowercase letters $(a, b, d \cdots)$ have different values in each paragraph. Besides, all the physical constants used in this paper are compiled in the appendix.

Through the following three paragraphs will apply the elementary algebra described above.

1. Quantum of action

Write the classical formulation for the undeterminancy principle of Heisenberg [2]

$$
\Delta q \times \Delta p \geq \hbar
$$

$\Delta q$ means the uncertainty in the position of a particle, while $\Delta p$ means the uncertainty in the momentum.

Will apply a discreteness view of the space together with a discreteness view of the momentum. Both specified in the following equation

$$
\left(N_{\ldots}\right)(o)\left(F_{\ldots}\right)=\hbar
$$

For purely practical reasons we have typed the arithmetical symbol $(=)$ instead of the symbol $(\geq)$.

$\hbar$ is the reduced Planck constant (appendix). As for the parameter $(o)$ it represents the value position. Consequently

$$
(o)=x \text { meters. }
$$

$\left(F_{\ldots}\right)$ is a sum of dics (discrete contribution series). Let's consider the following set of dics

$$
\begin{gathered}
a=\frac{1}{4}(1+2+16+32+16+2+1)=17.5 \\
b=\frac{a}{4^{2} 10^{2}}=0.0109375 \\
d=\frac{1}{4 \times 10^{4}}\left(16+8+1+\frac{1}{2}\right)=0.0006375 \\
e=\frac{1}{4 \times 10^{8}}(3+6+48+96)=0.00000153 .
\end{gathered}
$$

Compute the addition of the four series 


$$
\left(F_{\ldots}\right)=a+b+d+e=17.51157653 .
$$

Assign physical units

$$
\left(F_{\ldots}\right)=\left[M L T^{-1}\right]=17.51157653 \mathrm{~kg} \frac{\mathrm{m}}{\mathrm{s}} .
$$

On one side, we have the discrete distribution of $\left(N_{\ldots}\right)(o)$ possible positions of a particle and on the other hand we have a discrete distribution of velocities $\left(F_{\ldots}\right)$.

Will search, by means of one practical example, the algebraic product that is equal to the quantum of Action.

A particle with a mass equal to $10^{-31} \mathrm{~kg}$ moves at a speed (sum of discrete contributions of velocities) of $17.51157653 \times 10^{7} \frac{\mathrm{m}}{\mathrm{s}}$.

Set $(o)=\frac{1}{10^{35}} \mathrm{~m}$ same order of magnitude as Planck's length scale (13).

Apply such values in the equation

$$
\left(N_{\ldots}\right)(o)\left(F_{\ldots}\right)=\hbar
$$

which expressed in numbers results

$$
6.02214083 \times 10^{23} \times\left(\frac{1}{10^{35}} \mathrm{~m}\right) \times\left(10^{-31} \mathrm{~kg}\right) \times(17.51157653) \times 10^{7} \frac{\mathrm{m}}{\mathrm{s}}=\hbar .
$$

In words: the previous example describes the uncertainty in the position of a particle that is numerically equivalent to a discrete space consisting of $6.02214083 \times 10^{23}$ possible positions. Each position is $10^{-35}$ meters long. A particle with a mass equal to $10^{-31} \mathrm{~kg}$ moves at a distribution of velocities $\left(F_{\ldots}\right)$ (sum of discrete contributions of velocities) of $17.51157653 \times 10^{7} \frac{\mathrm{m}}{\mathrm{s}}$. After multiplying each of the terms the result is equal to the quantum of Action $\hbar$.

As for $\left(N_{\ldots}\right)=6.02214083 \times 10^{23}$ it computes as a dimensionless number. Despite having the same value as Avogadro's constant, it has nothing to do with it. ( $N_{\ldots}$ ) emerges algebraically from the relationship between $\left(F_{\ldots}\right),(o)$ and the reduced Planck constant $\hbar$.

Note that $6.02214083 \times 10^{23} \times\left(\frac{1}{10^{35}} \mathrm{~m}\right)=\left(N_{\ldots}\right) \times 10^{-12} \mathrm{~m}$, same order of magnitude as the Compton wavelength (Appendix).

Therefore the reduced Planck constant $\hbar$ acts as a conversion factor that determines the orders of magnitude assigned to the items $\left(F_{\ldots}\right),\left(N_{\ldots}\right)$ and $(o)$ located on the left side of the Equation (5).

2. Fine structure constant

Fine structure constant, or Sommerfeld constant, $\alpha$ (alpha) is a dimensionless number. The fine structure constant has long fascinated physicists. Attempts to find a mathematical origin have continued over time, and many scientists have proposed different developments looking for its ultimate meaning. From the physical point of view the fine structure constant characterizes the strength of the electromagnetic force, the interaction between electrically charged particles and light particles, The 2014 CODATA [3] recommended value, at zero energy is 


$$
\alpha=0,0072973525664 \cdots,
$$

whose standard uncertainty: $0.0000000017 \times 10^{-3}$.

Also the inverse value is usually used

$$
\alpha^{-1}=137.035999139 \cdots
$$

Standard uncertainty: 0.000000031 .

Consider an electrically charged particle whose rate of change of position in one second of time isn't unitary but the sum of an amount of rates of change of positions. It results from the interaction between the electrically charged particle and the photon per one second. Such interaction isn't considered unitary but complex. Experimental physics in the atomic and subatomic realm must arrange a lot of measurements. Under same conditions we can obtain different results. Therefore a statistical analysis are needed: calculate mean, typical deviation and probability.

Our aproach is merely arithmetical; based on the application of dics. Assume that the interactions between the charge and the quantum of Action are discrete. Natural numbers are involved. They translate a set of inputs of interactions per unit of time. For that all speed values are represented by a vector with the same direction but different magnitude.

Figure 1 represents grafically a set sketch of uncut, chaotic velocities. Whilst in Figure 2 we represent the sum of all speeds properly ordered by mean as of the dics application.

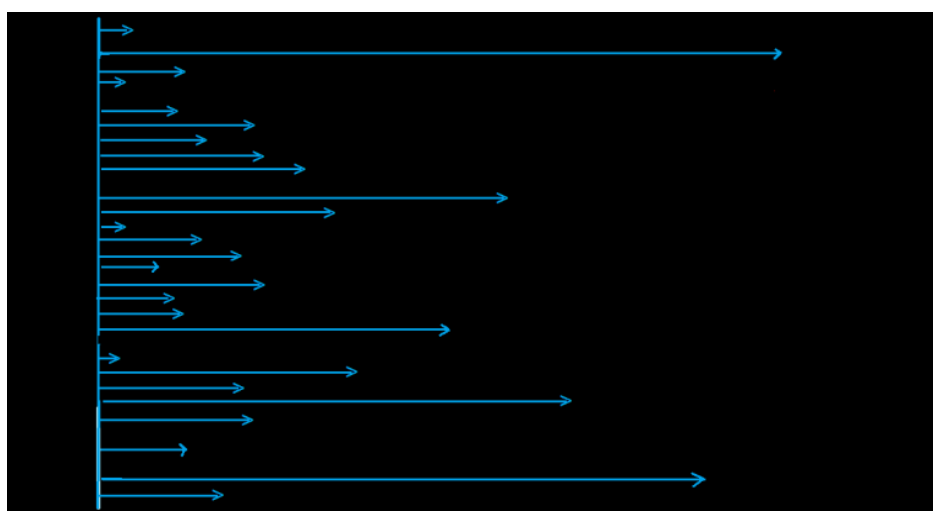

Figure 1. Disorderly or chaotic spectrum of velocities.

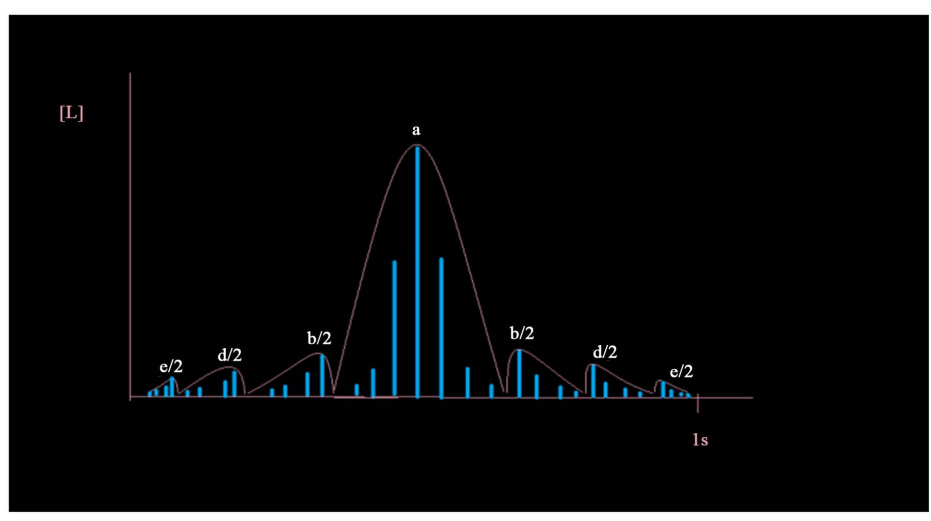

Figure 2. Spectrum of velocities properly ordered by means of dics application. 
Let's start writing the dics involved

$$
\begin{gathered}
a=(1+2+16+32+16+2+1) 10^{6}=70.000 .000 \\
b=(256+128+16+8) 10=4.080 \\
d=(128+64+8+4) 10=2.040 \\
e=(256+128+16+8) \frac{1}{10^{3}}=0.408
\end{gathered}
$$

Compute the sum of the four discrete contribution series typed above and assign speed units $\left[L T^{-1}\right]$

$$
F_{\ldots}=\frac{1}{32}(a+b+d+e)=2187691,26275 \frac{\mathrm{m}}{\mathrm{s}} .
$$

Photons (light particles) travels in the vacuum at the speed of light. Therefore will compute the ratio between the sum of dics (velocities ) and the speed of light in vacuum (Appendix).

$$
C=299.792 .458 \frac{\mathrm{m}}{\mathrm{s}}
$$

Finally write the ratio

$$
\frac{C}{F_{\ldots}}=\frac{1}{\alpha}=137,035999139 .
$$

Note that this value agrees exactly with all the significant digits, twelve significant figures, that defines the current accepted value of the inverse of the fine structure constant $\alpha^{-1}$.

3. Proton-electron ratio

In his book "The lightness of Being", Franck Wilczek [4] describes in a chapter the relationship between two equations (The music of the grid. A poem in two equations). On one side the second law of Einstein

$$
m=\frac{E}{c^{2}} .
$$

On the other hand the Planck-Einstein-Schroedinger formula

$$
E=h v \text {. }
$$

Both equations can be written as

$$
v=\frac{m c^{2}}{h}
$$

The symbol $v$ denotes the frequency mode of vibration. As for the mass of the proton and electron both are referred in the appendix.

Will apply this view to the proton-electron mass ratio using dics calculation (sketched in Figure 3).

$$
\frac{v_{p}}{v_{e}}=1836.153
$$

Let's apply the following series

$$
a=(128+64+8+4)
$$




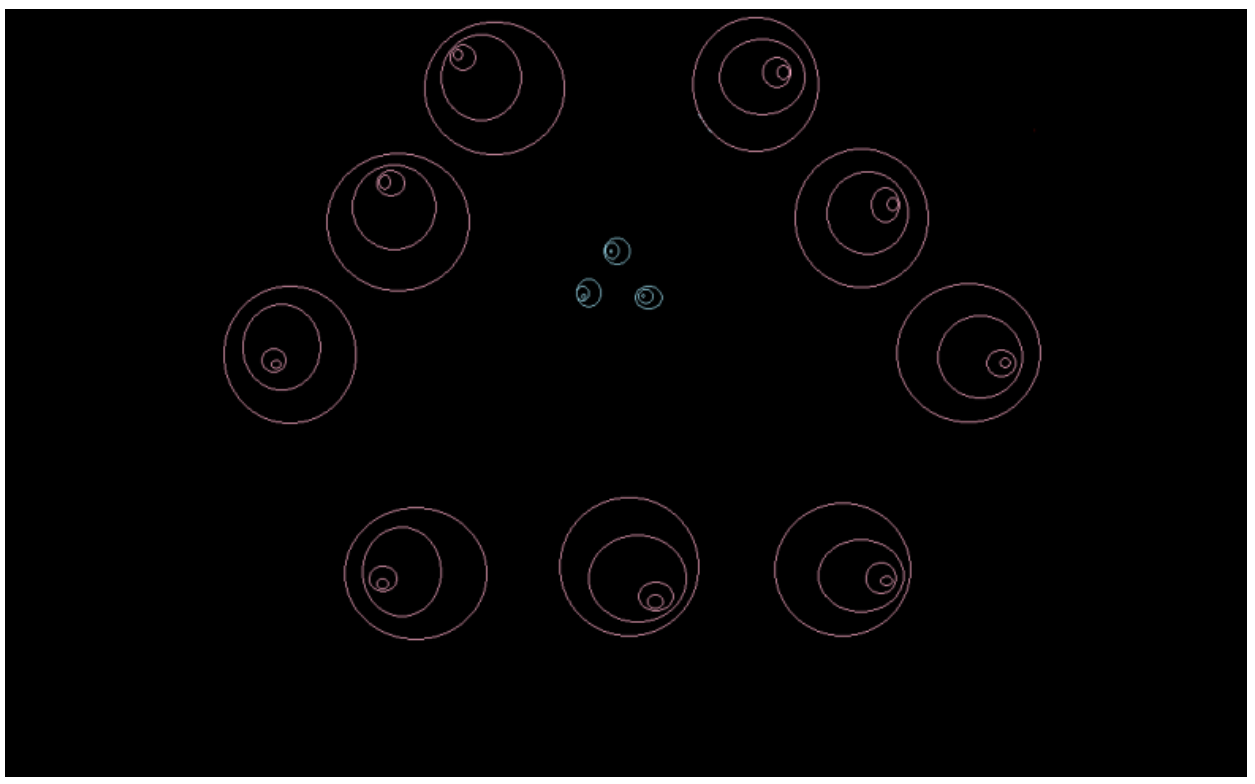

Figure 3. Proton-electron ratio.

$$
b=\frac{1}{10^{3}}(32+16+2+1)
$$

therefore

$$
\frac{v_{p}}{v_{e}}=9 a+3 b .
$$

\section{Discussion}

Since we are immersed in the theoretical approach of spacetime discreteness we went to search simple arithmetical tools. We find useful the application of a kind of series of natural numbers called dics. We have analyzed three physical topics.

Te value of the fine structure constant obtained by means of our method is the same that the current accepted value. The physical meaning is that the interaction, per unit of time between the charge of a particle and a photon isn't unitary but complex. Despite its complexity at first sight, is capable of analyzing. The tool that we have used is able to properly order the initial complexity.

Philosophy of discreteness has guided us when we develop a practical approach to the indeterminancy principle of Heisenberg. By means of the use of dics we have seen emerge one discreteness parameter associated to the discreteness of the space, i.e. the possible range of positions of a particle.

Finally we have applied a couple of simple series of natural numbers in the ratio between the proton mass and the electron mass. Figure 3 visually represents such topic. The sketch shows an ensemble of nine sets of large complex circles (9a in the equation 27) and a set of three small circles ( $3 b$ in the Equation (27)). By multiplying the frequency of vibration associated to the electron mass by $(9 a+3 b)$ we obtain the frequency of vibration associated to the mass of the proton. For now we do not want to go further into it. Just remember that a proton [5] is made of quarks and gluons. There are three quarks (uud) and there are three color-charge quantum states (White, red, blue) 
carried by each of the three quarks, resulting nine combinations of particles with the respective color charges.

\section{Conclusion}

We assume a spacetime discrete. Physical variables are considered as result of the sum of discrete contributions. Such discrete contributions can be calculated with the sum of natural numbers only. Experimental physics in the atomic and subatomic realm must arrange a lot of measurements. Our approach is merely arithmetical. Some sets of series of natural numbers are involved.

\section{References}

[1] Penrose, R. (2016) The Road to Reality: A Complete Guide to the Laws of the Universe. 63.

[2] Clark, A.D. (2014) Physics in Five Dimensions: Bye Bye Big Bang. 99.

[3] Mohr, P.J., et al. (2014) CODATA Recommended Values of the Fundamental Physical Constants.

[4] Wilczkek, F. (2009) The Lightness of Being. 133.

[5] L'Annunciata, M.F. (2016) Radioactivity: Introduction and History, From the Quantum to Quarks. 682.

[6] Mobilereference-2010-Tables of Universal, Electromagnetic, Atomic and Nuclear \& Physico-Chemical Constants.

\section{Appendix}

Table of physical constants [6]

Compton wavelength of the electron, $\lambda_{c}=2.42631 \times 10^{-12} \mathrm{~m}$

Planck constant, $h=6.62607 \times 10^{-34} \mathrm{Js}$

Reduced Planck constant, $\hbar=\frac{h}{2 \pi}=1.0545718 \times 10^{-34} \mathrm{Js}$

Speed of light in vacuum, $c: 299.792 .458 \frac{\mathrm{m}}{\mathrm{s}}$

Proton mass, $m_{p}: 1.67262 \times 10^{-27} \mathrm{~kg}$

Electron mass, $m_{e}: 9.109383 \times 10^{-31} \mathrm{~kg}$

Fine structure constant, $\alpha: 0.007297353$ 
Submit or recommend next manuscript to SCIRP and we will provide best service for you:

Accepting pre-submission inquiries through Email, Facebook, LinkedIn, Twitter, etc. A wide selection of journals (inclusive of 9 subjects, more than 200 journals)

Providing 24-hour high-quality service

User-friendly online submission system

Fair and swift peer-review system

Efficient typesetting and proofreading procedure

Display of the result of downloads and visits, as well as the number of cited articles Maximum dissemination of your research work

Submit your manuscript at: http://papersubmission.scirp.org/

Or contact jmp@scirp.org 